\title{
Analysis of Peanut Seed Oil by NIR
}

\author{
Babankumar Bansod', Ritula Thakur'2, Ronald Holser ${ }^{3}$ \\ ${ }^{1}$ Agrionics, CSIR-CSIO, Chandigarh, India \\ ${ }^{2}$ Electrical Engineering Department, NITTTR, Chandigarh, India \\ ${ }^{3}$ Russell Research Center, USDA-ARS, Athens, USA \\ Email: Ronald.Holser@ars.usda.gov
}

Received 18 July 2015; accepted 6 November 2015; published 9 November 2015

Copyright @ 2015 by authors and Scientific Research Publishing Inc.

This work is licensed under the Creative Commons Attribution International License (CC BY). http://creativecommons.org/licenses/by/4.0/

(c) (i) Open Access

\begin{abstract}
Near infrared reflectance spectra (NIRS) was collected from Arachis hypogaea seed samples and used in predictive models to rapidly identify varieties with high oleic acid. The method was developed for shelled peanut seeds with intact testa. Spectra was evaluated initially by principal component analysis (PCA) followed by partial least squares (PLS). PCA performed with full spectra and reduced spectra with one principal component accounted for $97 \%$ to $99 \%$ variability, respectively. The PLS model generated from first derivative spectra provided a standard error of prediction (SEP) of 7.7204808. This technique provides a non-destructive method to rapidly identify high oleic peanut seeds to support the selection and cultivation of high oleic acid peanut varieties. The method can also be useful at peanut processing facilities for screening and quality assessments.
\end{abstract}

\section{Keywords}

Chemometrics, Infrared Spectroscopy, Oilseed, Oleic Acid, Quality Control

\section{Introduction}

Near infrared reflectance spectroscopy (NIRS) is widely used by the food industry for routine oil, protein, and moisture analysis. It is expanding into agricultural applications with the development of precision farming techniques. This includes both crop production and post-harvest applications. The infrared instrumentation developed for laboratory use is now commercially available in smaller and more rugged designs suitable for use in field and processing facility applications. These new devices are versatile and may be incorporated with remote sensing equipment or integrated with process control systems.

Interest in high oleic peanut varieties has increased recently due to the health benefits associated with unsaturated fatty acids [1] [2]. The analysis of oilseed lipids by spectroscopic methods demonstrated great success and 
was accepted as the basis for AOCS standard methods [3]. The standard method focused on the measurement of total oil although the spectroscopic approach was also applied to determine fatty acid profiles and lipid oxidation products [4] [5]. The technique proved useful for quality assessments in food and feed formulations supplemented with polyunsaturated fatty acids such as docosahexaenoic acid and linolenic acid. These compounds are particularly susceptible to oxidation and the formation of malodorous degradation products [6] [7]. Advantages of infrared spectroscopic analysis include minimal sample preparation and reduced analysis time compared to other methods [8] [9].

The combination of spectroscopic and chemometric methods provides a powerful technique to detect, interpret, and model changes in spectra correlated with sample composition. These results may be used both for descriptive and predictive purposes. Spectra are collected and subjected to various pre-processing transformations to facilitate analysis and model development. Different spectral regions carry information of greater interest to a particular problem. A systematic approach to test and validate a predictive model is necessary. This investigation used two of the most common chemometric techniques, principal component analysis (PCA) and partial least squares (PLS), to analyze a set of near infrared spectra obtained from shelled peanuts with varying amounts of oleic acid.

\section{Experimental}

\subsection{Materials and Methods}

Peanut seed samples of normal and high oleic varieties were provided by Prof. Naveen Puppala, New Mexico State University, NM, USA. Peanuts were shelled and stored in a -5 C freezer until measurements were taken. Extraction and analysis of peanut lipids were performed by gas chromatography following AOCS standard method [10].

\subsection{Spectroscopy}

Near infrared reflectance spectra were collected with the SeedMeister model 709A spectrophotometer manufactured by the Brimrose Corp. of America, Sparks, MD, USA. Samples were scanned from 1200 to $2000 \mathrm{~nm}$. Spectral analyses were performed with Unscrambler X, Camo Software, Oslo, Norway. Spectra were examined initially by principal component analysis (PCA) to identify clusters of high oleic and normal oleic content seeds. PCA is useful to construct models that describe data. PCA transforms the original variables into new variables that are linear combinations of the original variables and calculated to reduce the variability in the data [11] [12]. Spectra were transformed by multiplicative scatter correction (MSC) and first derivative Norris-gap. After the descriptive PCA models were evaluated the predictive partial least squares (PLS) models were developed [13]. PLS attempts to converge the model to a minimum residual error condition. The resulting model may be used to describe spectral data, however, in this case it was used to predict values of oleic acid from spectral data. The error between measured and predicted values is described by either the root mean square error of prediction (RMSEP) or the standard error of prediction (SEP) [14]. PLS models were generated from full spectra, 1200 $2000 \mathrm{~nm}$, and reduced spectra, 1600 - $1800 \mathrm{~nm}$. Analyses were performed with cross-validation using 8 segments and 1225 calibration samples. Model performance was reported in terms of standard error of prediction (SEP).

\section{Results and Discussion}

The infrared spectra from normal and high oleic peanut seeds are shown in Figure 1. These curves are averages of fifteen first derivative spectra. The spectral features appear very similar and require chemometric analysis to identify differences that may correlate to changes in seed oil compositions. Evaluation of the spectral data was performed by principal component analysis (PCA) to visualize groups or clusters of data and reduce the number of variables, i.e., wavelengths, required to develop a predictive model. The application of PCA provided a descriptive model to explore the spectral data and estimate the success of a predictive model. If PCA did not show two groups that correlated to normal and high oleic content oil seeds no predictive model could be developed.

Initial results from PCA are presented in Figure 2 and Figure 3 using full and reduced spectra, respectively. These plots show two varieties of peanut seeds, e. g., normal oleic and high oleic content. In Figure 2 the full spectral range of 1200 - $2000 \mathrm{~nm}$ was used and the first principal component accounted for $97 \%$ of the variabil- 


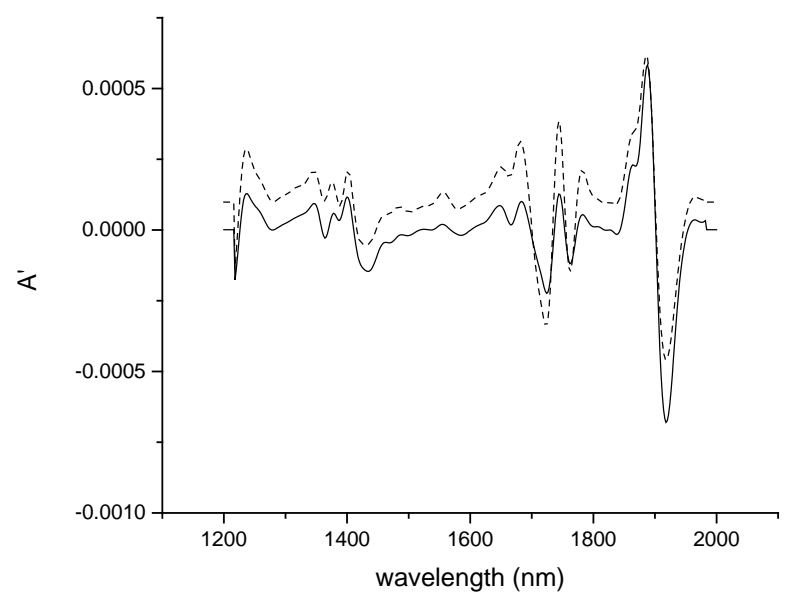

Figure 1. Infrared derivative spectra of normal (dash) and high (solid) oleic peanut seeds.

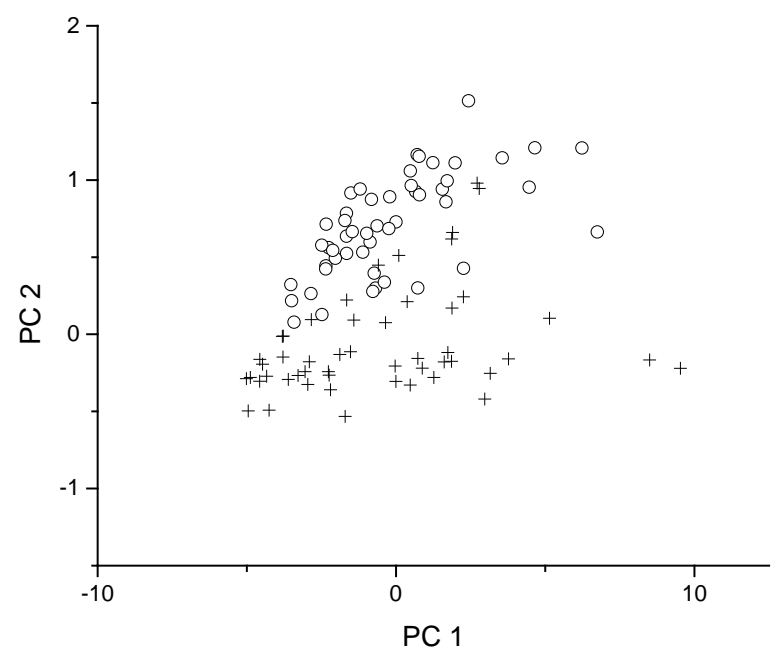

Figure 2. PCA score plots for normal (o) and high (+) oleic acid seeds. Spectra range: $1200-2000 \mathrm{~nm}$.

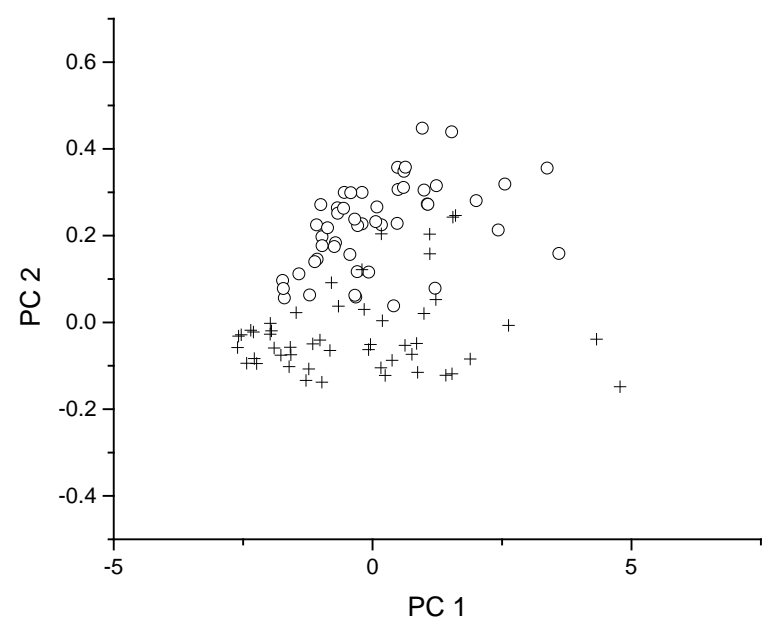

Figure 3. PCA score plots for normal (o) and high (+) oleic acid seeds. Spectra range: $1600-1800 \mathrm{~nm}$. 
ity in the measured values. In Figure 3 the PCA results obtained for the reduced spectral region of 1600 - 1800 $\mathrm{nm}$ accounted for $99 \%$ of the variability with one principal component. In both cases there is separation into two groups corresponding to normal oleic and high oleic oilseeds. These results show the potential for successfully developing models to predict oleic acid content of shelled peanut seeds from infrared spectra. At this point the descriptive PCA model has indicated very good probability to construct a predictive model using a regression technique such as partial least squares (PLS).

Subsequent analysis by PLS was performed with the first derivative spectra and provided the results shown in Figure 4 and Figure 5 for predictive models based on the full and reduced spectral regions. Figure 4 displays the score plots for the full spectra case with $64 \%$ of the variability in the predicted value accounted by the first factor. The score plot for the model developed from the reduced spectral region in Figure 5 accounted for $98 \%$ of the variability in the predicted value by the first factor. Models for both cases are able to predict normal and high oleic peanut seeds. The performance of the two models is described in more detail by the standard error of prediction (SEP) values. Results for the two models described by the first and second factors showed the SEP value decreased from 12.08858 for the full model to 7.204808 for the reduced model. This represents a significant improvement in the ability of the reduced model to accurately predict oleic acid in peanuts. The SEP carries the units of the predicted variable, \% oleic acid, and may be interpreted accordingly. For example, if both models predicted $70 \%$ oleic acid content the error in this value would be $12.08858 \%$ for the full model and $7.204808 \%$ for the reduced model.

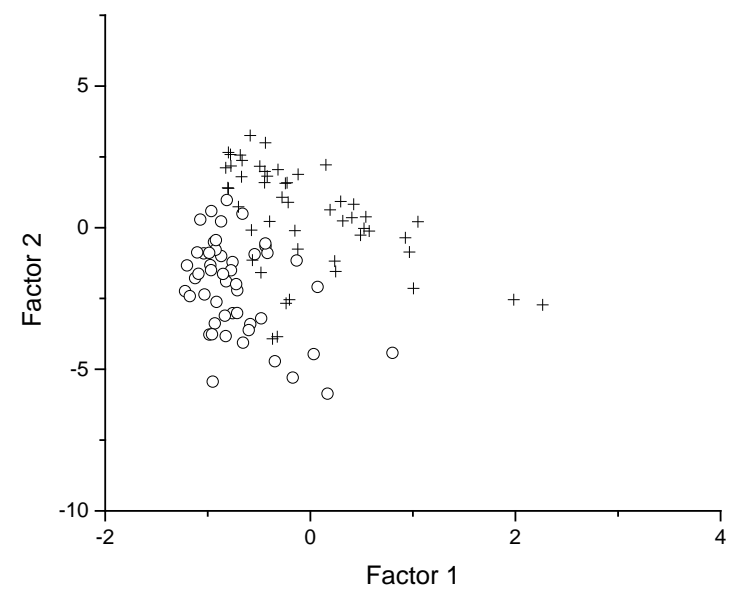

Figure 4. PLS scores plot for normal (o) and high (+) oleic acid seeds. Spectra range: $1200-2000 \mathrm{~nm}$.

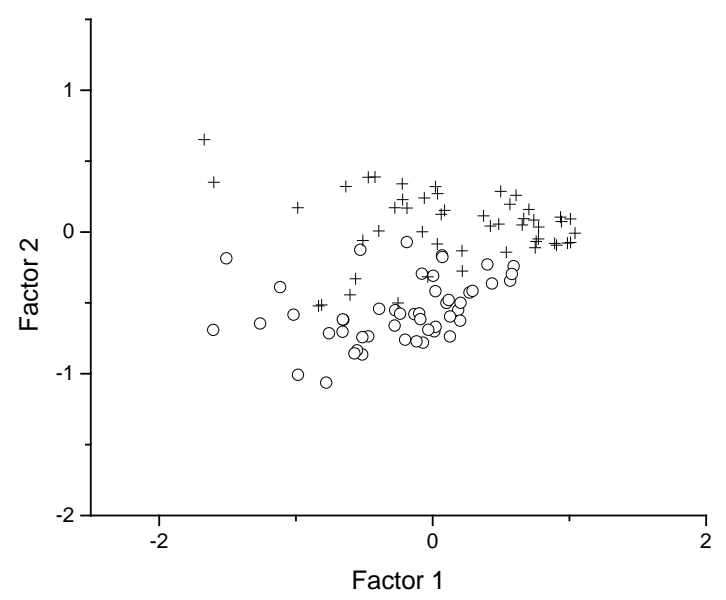

Figure 5. PLS scores plot for normal (o) and high (+) oleic acid seeds. Spectra range: 1600 - $1800 \mathrm{~nm}$. 
Further refinement of the model by reduction of the spectral region used is possible using techniques such as two-dimensional correlation spectroscopy and multivariate curve resolution [15] [16]. These methods provide a systematic approach to select spectral regions, reduce the number of calculations, and improve the predictive capability of the model [17] [18]. However, the current model is adequate for the determination of normal and high oleic peanut seeds without additional modifications.

\section{Conclusion}

The importance of selecting and cultivating high oleic peanut varieties is motivated by the health benefits associated with seed oil composed of stable unsaturated fatty acids such as oleic acid. NIRS provides a tool capable of rapidly and non-destructively measuring the amount of oleic acid in shelled peanut seeds. The implementation of predictive models used with modern infrared instrumentation supports this objective and finds applications in the field, the laboratory, and peanut processing facilities.

\section{Acknowledgements}

The authors are grateful to Dr. Naveen Puppala for guidance on this project.

\section{References}

[1] Leaf, A. (1990) Cardiovascular Effects of Fish Oils. Beyond the Platelet. Circulation, 82, 624-628. http://dx.doi.org/10.1161/01.CIR.82.2.624

[2] Tapiero, H., Nguyen Ba, G., Couvreur, P. and Tew, K.D. (2002) Polyunsaturated Fatty Acids (PUFA) and Eicosanoids in Human Health and Pathologies. Biomedicine and Pharmacotherapy, 56, 215-222. http://dx.doi.org/10.1016/S0753-3322(02)00193-2

[3] Method Cd 14d-99 (2009) Rapid Determination of Isolated Trans Geometric Isomers in Fats and Oils by Attenuated Total Reflectance Spectroscopy. Official Methods and Practices of the AOCS, AOCS Press, Urbana, 1-6.

[4] Sundaram, J., Kandala, C., Holser, R., Butts, C. and Windham, W. (2010) Determination of In-shell Peanut Oil and Fatty Acid Composition Using Near-Infrared Reflectance Spectroscopy. Journal of the American Oil Chemists' Society, 87, 1103-1114. http://dx.doi.org/10.1007/s11746-010-1589-7

[5] Holser, R. and Hawkins, S. (2011) Measurement of Lipid Supplements in Poultry Feed by Infrared Spectroscopy. International Journal of Poultry Science, 10, 677-679. http://dx.doi.org/10.3923/ijps.2011.677.679

[6] Wood, J.D., Richardson, R.I., Nute, G.R., Fisher, A.V., Campo, M.M., Kasapidou, E., Sheard, P.R. and Enser, M. (2004) Effects of Fatty Acids on Meat Quality: A Review. Meat Science, 66, 21-32. http://dx.doi.org/10.1016/S0309-1740(03)00022-6

[7] Schauenstein, E. (1967) Autoxidation of Polyunsaturated Esters in Water: Chemical Structure and Biological Activity of the Products. Journal of Lipid Research, 8, 417-428.

[8] Kawai, Y., Takeda, S. and Terao, J. (2007) Lipidomic Analysis for Lipid Peroxidation-Derived Aldehydes Using Gas Chromatography-Mass Spectrometry. Chemical Research in Toxicology, 20, 99-107.

http://dx.doi.org/10.1021/tx060199e

[9] Esterbauer, H. and Zollner, H. (1989) Methods for Determination of Aldehydic Lipid Peroxidation Products. Free Radical Biology and Medicine, 7, 197-203. http://dx.doi.org/10.1016/0891-5849(89)90015-4

[10] Method Ce 1j-07 (2009) Determination of Cis-, Trans-, Saturated, Monosaturated, and Polyunsaturated Fatty Acids in Extracted Fats by Capillary GLC. Official Methods and Practices of the AOCS, AOCS Press, Urbana, 1-50.

[11] Darton, R. (1980) Rotation in Factor Analysis. The Statistician, 29, 167-194. http://dx.doi.org/10.2307/2988040

[12] Hotelling, H. (1933) Analysis of a Complex of Statistical Variables into Principal Components. Journal of Educational Psychology, 24, 417-441, 498-520.

[13] Dayal, B. and MacGregor, J. (1997) Improved PLS Algorithms. Journal of Chemometrics, 11, 73-85. http://dx.doi.org/10.1002/(SICI)1099-128X(199701)11:1<73::AID-CEM435>3.0.CO;2-\#

[14] Lindgren, F., Geladi, P. and Wold, S. (1993) The Kernel Algorithm for PLS. Journal of Chemometrics, 7, 45-59. http://dx.doi.org/10.1002/cem.1180070104

[15] Sasic, S., Morimoto, M., Otsuka, M. and Ozaki, Y. (2005) Two-Dimensional Correlation Spectroscopy as a Tool for Analyzing Vibrational Images. Vibrational Spectroscopy, 37, 217-224. http://dx.doi.org/10.1016/j.vibspec.2004.09.005

[16] Muika, B., Lendl, B., Molina-Diaz, A., Valcarcel, M. and Ayora-Canada, M.J. (2007) Two-Dimensional Correlation Spectroscopy and Multivariate Curve Resolution for the Study of Lipid Oxidation in Edible Oils Monitored by FTIR 
and FT-Raman Spectroscopy. Analytica Chimica Acta, 593, 54-67. http://dx.doi.org/10.1016/j.aca.2007.04.050

[17] Himmelsbach, D. and Holser, R. (2009) Application of 2D Correlation Spectroscopy with MCR in the Preparation of Glycerol Polyesters. Vibrational Spectroscopy, 51, 142-145. http://dx.doi.org/10.1016/j.vibspec.2009.03.004

[18] Noda, I. (2008) Recent Advancement in the Field of Two-Dimensional Correlation Spectroscopy. Journal of Molecular Structure, 883/884, 2-26. http://dx.doi.org/10.1016/j.molstruc.2007.11.038 